\title{
Capsule Commentary on Liss et al., Specialty Use Among Patients with Treated Hypertension in a Patient-Centered Medical Home
}

\author{
Heather Klusaritz, PhD, MSW',2, Giang Nguyen, MD, MSCE, MPH, FAAFP ${ }^{1,2,3}$, and Peter F. Cronholm, \\ $M D, M S C E, F A A F P^{1,2,3}$ \\ 'Department of Family Medicine and Community Health, University of Pennsylvania, Philadelphia, PA, USA; ${ }^{2}$ Center for Public Health Initiatives, \\ University of Pennsylvania, Philadelphia, PA, USA; ${ }^{3}$ Leonard Davis Institute of Health Economics, University of Pennsylvania, Philadelphia, PA, USA.
}

J Gen Intern Med 29(5):786

DOI: $10.1007 / \mathrm{s} 11606-014-2824-y$

(c) Society of General Internal Medicine 2014

$\mathrm{H}$ eather Klusaritz, PhD, MSW

While the patient-centered medical home (PCMH) continues to gain momentum as a transformative primary care delivery model, the impact on utilization patterns between primary and specialty care providers is not well understood. Ideally, patients receiving primary care in a PCMH would shift utilization from outpatient specialty to primary care providers for medical needs other than care of complex or rare conditions. Liss et al. examined specialty care utilization of hypertensive patients in an integrated care system undergoing PCMH practice transformation. ${ }^{1}$ They found decreased specialty care utilization following $\mathrm{PCMH}$ transformation for all but the highest morbidity patient groups. These findings offer an important first step in understanding the interplay between primary and specialty care utilization in healthcare systems adopting the PCMH model. However, the study population was almost entirely insured through commercial plans or Medicare, with only $1.3 \%$ insured through Medicaid or state-subsidized insurance.

Utilization patterns of Medicaid patient populations may not be as sensitive to the PCMH care model. Medicaid patients face a greater burden of access barriers and life stressors, potentially inhibiting engagement with systems of care. Further, practices that primarily serve a Medicaid population face more complex psychosocial demands that may drain energy away from PCMH activities. Finally, research suggests different care preferences for Medicaid populations; patients with low socioeconomic status may preferentially seek hospital-based specialist care over ambulatory care due to perceptions about quality of care. ${ }^{2}$ Given that Medicaid patients already have limited access to specialists, ${ }^{3,4}$ patients from these populations may also be resistant to efforts shifting them away from specialty care related to historical concerns about denied access and further marginalization. The process of PCMH transformation in practices with high Medicaid rates will need to be sensitive to historical factors shaping patient care utilization preferences when shifting care from specialists where appropriate, and focus efforts on trust-building with at-risk populations. Future research studying the impact of PCMH transformation on specialty service utilization will need to focus further on potential differential effects in Medicaid patient populations.

Conflict of Interest: The authors have no conflicts of interest with this article.

Corresponding Author: Heather Klusaritz, PhD, MSW; Department of Family Medicine and Community HealthUniversity of Pennsylvania, Philadelphia, PA, USA (e-mail: Heather.Klusaritz@uphs.upenn.edu).

\section{REFERENCES}

1. Liss DT, Fishman PA, Rutter CM, Grembowski D, Ross TR, Reid RJ. Specialty Use among Patients with Treated Hypertension in a PatientCentered Medical Home. J Gen Intern Med. doi: 10.1007/s11606-0142776-2.

2. Kangovi S, Barg FK, Carter T, Long JA, Shannon R, Grande D. Understanding why patients of low socioeconomic status prefer hospitals over ambulatory care. Health Aff. 2013;32(7):1196-1203. doi:10.1377/ hlthaff.2012.0825.

3. Burns ME. Medicaid managed care and health care access for adult beneficiaries with disabilities. Health Serv Res. 2009;44(5 Pt 1):1521-41. doi:10.1111/j.1475-6773.2009.00991.x.

4. Rhodes KV, Bisgaier J, Lawson CC, Soglin D, Krug S, Van Haitsma M. Patients who can't get an appointment go to the ER: access to specialty care for publicly insured children. Ann Emerg Med. 2013;61(4):394-403. doi:10.1016/j.annemergmed.2012.10.030. 